\title{
Erratum to: Enterprise Microblogging
}

DOI 10.1007/s12599-010-0137-1

\section{The Authors}

PD Dr. Kai Riemer ( $₫)$

Discipline of Business Information

Systems

Faculty of Economics \& Business

University of Sydney

Building $\mathrm{H} 69$

Sydney

NSW 2006

Australia

kai.riemer@sydney.edu.au

\section{Dr. Alexander Richter}

Institut für Softwaretechnologie

Fakultät für Informatik

Universität der Bundeswehr München

85577 Neubiberg

Germany

a.richter@unibw.de

Dipl.-Wirt.-Inf. Martin Böhringer Professur für Wirtschaftsinformatik II, insb. Systementwicklung/

Anwendungssysteme

Fakultät für Wirtschaftswissenschaften Technische Universität Chemnitz 09126 Chemnitz

Germany

martin.boehringer@wirtschaft.

tu-chemnitz.de

Published online: 2010-12-09

The online version of the original article can be found under doi: 10.1007/s12599-010-0129-1.

(c) Gabler Verlag 2010
Bus Inf Syst Eng (2010) 2(6): 391-394

The family name of author Böhringer was, unfortunately, not published correctly in the article. The correct name is: Dipl.-Wirt.-Inf. Martin Böhringer (not Bohringer). 